\title{
Non-invasive detection of gastro-oesophageal reflux using an ambulatory system
}

\author{
N Washington, H A Moss, C Washington, J L Greaves, R J C Steele, C G Wilson
}

\begin{abstract}
The aim of this study was the separate measurement of reflux of food and acid into the oesophagus in 37 healthy, ambulant subjects. This was performed by radiolabelling the food and monitoring its reflux with a small directional gamma detector, which was placed externally over the oesophagus, and connected to an ambulatory data recorder. The $\mathrm{pH}$ was measured with a conventional oesophageal pH electrode. This method permitted the separate characterisation of acid and neutral (food) components of gastro-oesophageal reflux. The gastric emptying characteristics of the test meal were also monitored by gamma scintigraphy in a separate experiment. The oesophageal pH fell below 4 for $3 \cdot 2+8 \cdot 6 /-2 \cdot 3 \%$ (mean (SD)) of the recording time. Food reflux alone occurred for $17 \cdot 8+53 \cdot 2 /-13 \cdot 8 \%$ of the recording time. Simultaneous food and acid reflux occurred for only $0 \cdot 95+5 \cdot 2 /-1 \cdot 2 \%$ of the time. Not every reflux event detected by a fall in $\mathbf{p H}$ was seen as an increase in counts as a result of reflux of food, and vice versa. This poor correlation of food and acid reflux implies incomplete mixing of food and acid in the stomach, and further shows the inadequacy of reflux diagnosis methods that depend on $\mathrm{pH}$ detection alone.

(Gut 1993; 34: 1482-1486)
\end{abstract}

Ambulatory $\mathrm{pH}$ monitoring is a well established technique for the assessment of gastrooesophageal reflux. Various parameters are used to assess the severity of reflux, for example, percentage time for which the oesophagus reaches a pH of less than 4 , number of episodes, and average duration of each reflux episode. ${ }^{1}$ Although these data are extremely useful, it cannot assess reflux of potentially harmful materials such as bile, which has a near neutral $\mathrm{pH}$, or even food with a near neutral $\mathrm{pH}$ that may contain other damaging agents such as pepsin. Reflux of radiolabelled food and liquids can be measured using the technique of gamma scintigraphy ${ }^{23}$; however, in this procedure the subjects are non-ambulant as they have to be continually monitored by standing in front of, or lying under, a gamma camera to detect the reflux event. It is therefore not practical to monitor patients for long periods with this technique. Also reflux provoked by postural changes such as stooping cannot be measured by this method. Various techniques to promote reflux while imaging have been attempted, such as abdominal cuffing, in which a binder is placed over the abdomen and the pressure is increased by inflating it with air. ${ }^{4}$ It has been argued that compression of the abdomen increases the 'pinchcock' effect of the angular incisura and so it is possible that this manoeuvre could actually prevent reflux in some patients.

Gamma scintigraphy has been shown to be more sensitive than either radiography or the standard acid reflux test in the detection of gastro-oesophageal reflux, ${ }^{2}$ with a sensitivity of $85.5 \%$, compared with $69.5 \%$ with the standard acid reflux test and $27 \cdot 5 \%$ with radiography. Scintigraphy is also a sensitive test in patients where endoscopy and histological examination cannot confirm the presence or absence of reflux. ${ }^{5}$ The advantages of gamma scintigraphy are offset by the fact that patients are required to be static during imaging, whereas it has been shown that it is more reliable to monitor reflux in ambulant patients than patients restricted within a hospital routine. ${ }^{6}$

At Nottingham we have developed a technique for measuring reflux of radiolabelled food into the oesophagus in ambulant subjects ${ }^{7}$ using a highly collimated cadmium telluride gamma detector, which is worn in a harness over the oesophagus. To compare the new technique with the conventional intraoesophageal $\mathrm{pH}$ measurement, a method was developed to locate a $\mathrm{pH}$ electrode at the same point in the oesophagus as that monitored by the cadmium telluride detector. The reflux patterns of both food and acid were then measured in 37 healthy volunteer subjects after a refluxogenic meal. The gastric residence of the solid and liquid phases of the meal in the stomach was also characterised using gamma scintigraphy in healthy volunteers. The gastric $\mathrm{pH}$ profile produced by the meal was also measured in the same subjects and its total buffering capacity was measured in vitro by titration.

\section{Methods}

The test meal consisted of (ingredients per person): two eggs (approximate weight $120 \mathrm{~g}$ ), $50 \mathrm{~g}$ grated cheese, $30 \mathrm{~g}$ each of tomato, onion, mushroom, green peppers, and smoked mackerel, $10 \mathrm{~g}$ butter, one slice buttered toast, $250 \mathrm{ml}$ lemonade, one small (about $50 \mathrm{~g}$ ) apple pie, $50 \mathrm{~g}$ ice cream, and one cup of coffee (with cream and sugar if desired). The meal was served as a savoury omelette with the lemonade, followed by apple pie and ice cream with a cup of coffee as a second course. Identical test meals were used for all the experiments. This meal was designed to include many reflux provoking foods to maximise postprandial reflux in normal subjects.

IN VITRO STUDIES

Buffering capacity of the test meal

The cooked test meal was cut into small pieces, 
mixed thoroughly, and placed in a beaker in a water bath at $37^{\circ} \mathrm{C}$. One hundred millilitre samples of simulated gastric juice (USP formulation) were then added to the test meal, the mixture was stirred thoroughly, and the resultant $\mathrm{pH}$ measured when it had stabilised after 10 minutes. Additions were repeated until a $\mathrm{pH}$ of 2 was obtained.

\section{Characterisation of the collimation for the gamma detector}

The experiments required the gamma detector (Bionuclear Services Ltd, Cornwall) to be well shielded from the activity in the stomach while still being able to detect the potentially small amount of refluxed activity in the oesophagus. The polar and cartesian responses of the detector were measured with a small source of technetium- $99 \mathrm{~m}\left({ }^{99 m} \mathrm{Tc}\right)$. The shielding with the collimator supplied by the manufacturers was insufficient as it caused a high background level, and hence a new lead collimator was designed to provide a more directional response.

\section{IN VIVO STUDIES}

\section{Ethical considerations arid volunteer selection}

Healthy male and non-pregnant female volunteers, age range 19 to 31 , were recruited from the University student population. Subjects were screened by a fully qualified medical practitioner. Female subjects were tested for pregnancy on the morning of the trial. The volunteers were given both written and verbal information as to the nature of the trial. Approval was obtained from the Nottingham University Hospital Ethical Committee. Permission to give isotopes was obtained from the Department of Health.

\section{Measurement of the gastric emptying of liquid and solid phases of the test meal}

The omelette was labelled by the addition of $2 \mathrm{MBq}$ technetium-99m $\left({ }^{99 \mathrm{~m}} \mathrm{Tc}\right)$ tin colloid per subject into the eggs before cooking. The integrity of this method of labelling the meal has been well established by incubation of labelled scrambled egg with simulated gastric juice at $37^{\circ} \mathrm{C}$ and monitoring the rate of release of the label into the liquid phase. The release of the label into the liquid phase correlated with the digestion of the egg by the pepsin. ${ }^{8-10}$ In addition the liquid phase of the meal was labelled by the addition of $2 \mathrm{MBq}$ Indium-113m diethylenetriaminepentaacetic acid per subject ( ${ }^{113 m}$ In DTPA) to the lemonade.

Six healthy female subjects were fasted overnight, and on the morning of the trial ingested the radiolabelled test meal. The gamma camera (GEC Maxicamera II) was fitted with a high energy parallel hole collimator $(400 \mathrm{keV}$ maximum energy). Anterior and posterior images of 30 seconds' duration were recorded at 15 minute intervals starting immediately after the subjects had ingested the first course of the meal. Imaging was continued until the meal had left the stomach. Radioactive anterior and posterior markers were placed on the thorax opposite the stomach to permit accurate alignment of subsequent images. The ${ }^{99 \mathrm{~m}} \mathrm{Tc}$ and ${ }^{113 \mathrm{~m}} \mathrm{In}$ images were recorded simultaneously, but stored separately on the computer for subsequent analysis. The counts were corrected for background contribution, radioactive decay, and energy overlap. The geometric mean of the anterior and posterior counts was calculated to correct for tissue attenuation. Interpolated data from each subject were then used to calculate the mean gastric emptying curves.

\section{Measurement of the $\mathrm{pH}$ in the fundus after the test} meal

The pH probe (Radiometer, Copenhagen) was sterilised and calibrated immediately before and after use. The probe was labelled with approximately $0.5 \mathrm{MBq}$ of technetium dried onto a small square of filter paper, sealed under waterproof tape, and placed very close to the electrode tip.

The gastric $\mathrm{pH}$ was measured in the same six volunteers who had participated in the gastric emptying study. The volunteers fasted overnight, or for six hours after a light breakfast if the study was carried out in the afternoon. The $\mathrm{pH}$ probe was passed nasogastrically with a small amount of water. While standing in front of the gamma camera, the subject sipped a small amount of ${ }^{99 \mathrm{~m}}$ Tc-DTPA labelled water to outline the stomach. The $\mathrm{pH}$ probe was then positioned in the fundus $5 \mathrm{~cm}$ below the cardia.

The $\mathrm{pH}$ was recorded at 15 second intervals by a solid state ambulatory recorder (Novo Memolog, Vertec Scientific, Reading). Baseline values were recorded for approximately 15 minutes. The meal was then ingested and the $\mathrm{pH}$ values were recorded for approximately four hours. At the end of the study period, the information was transferred from the Memolog recorder into an Apple Macintosh computer for analysis.

The $\mathrm{pH}$ values for each subject were converted to $\mathrm{H}^{+}$concentrations. These were averaged for the six subjects and the SEM was calculated. These values were then converted back to the $\mathrm{pH}$ scale. This provided an arithmetic mean of the $\mathrm{H}^{+}$concentration, rather than a geometric mean, which is calculated by averaging the $\mathrm{pH}$ values directly.

\section{pH Monitoring and food reflux detection study} The study was carried out in 21 male and 16 female volunteers. The test meal was radiolabelled with $1 \mathrm{MBq}{ }^{99 \mathrm{~m}} \mathrm{Tc}$ tin colloid as described earlier, and in addition $1 \mathrm{MBq}^{99 \mathrm{~m}} \mathrm{Tc}$ DTPA per subject was added to the lemonade. One isotope was used to label both phases of the meal because the gamma detector cannot discriminate between isotopes. The $\mathrm{pH}$ probe was prepared and marked as before but the radioactive marker was placed $5 \mathrm{~cm}$ from the electrode tip to be out of view of the gamma detector.

The volunteers fasted overnight or for six hours after a light breakfast if the study was carried out in the afternoon. The $\mathrm{pH}$ probe was passed either nasogastrically or orogastrically with a small amount of water. Fifty $\mathrm{ml}$ of ${ }^{99 \mathrm{~m}} \mathrm{Tc}$ - 
DTPA labelled water (activity of $0.5 \mathrm{MBq}$ ) was given to the subject while they were standing in front of the gamma camera to outline the oesophagus and stomach. The $\mathrm{pH}$ probe was then positioned in the oesophagus with the radioactive tag $10 \mathrm{~cm}$ from the cardia using the gamma camera, thus positioning the tip $5 \mathrm{~cm}$ above the cardia. The position of the gamma detector was identified by moving a small sealed ${ }^{99 \mathrm{~m}} \mathrm{Tc}$ source over the chest with the subject standing in front of the gamma camera. The source was positioned $5 \mathrm{~cm}$ above the cardia and $5 \mathrm{~cm}$ below the tag on the $\mathrm{pH}$ probe. The exterior source was then removed and replaced with the gamma detector, which was worn in a harness to fix its position. Both the gamma detector and the $\mathrm{pH}$ probe were connected to a dual channel solid state recorder (Novo Memolog 600 system, Vertec Scientific, Reading). The timebase was set to record at 15 second intervals. Subjects then ingested the radiolabelled meal. Recording of data took place for approximately four hours, while the subject was ambulant; at the end of this period, the $\mathrm{pH}$ probe and gamma detector were

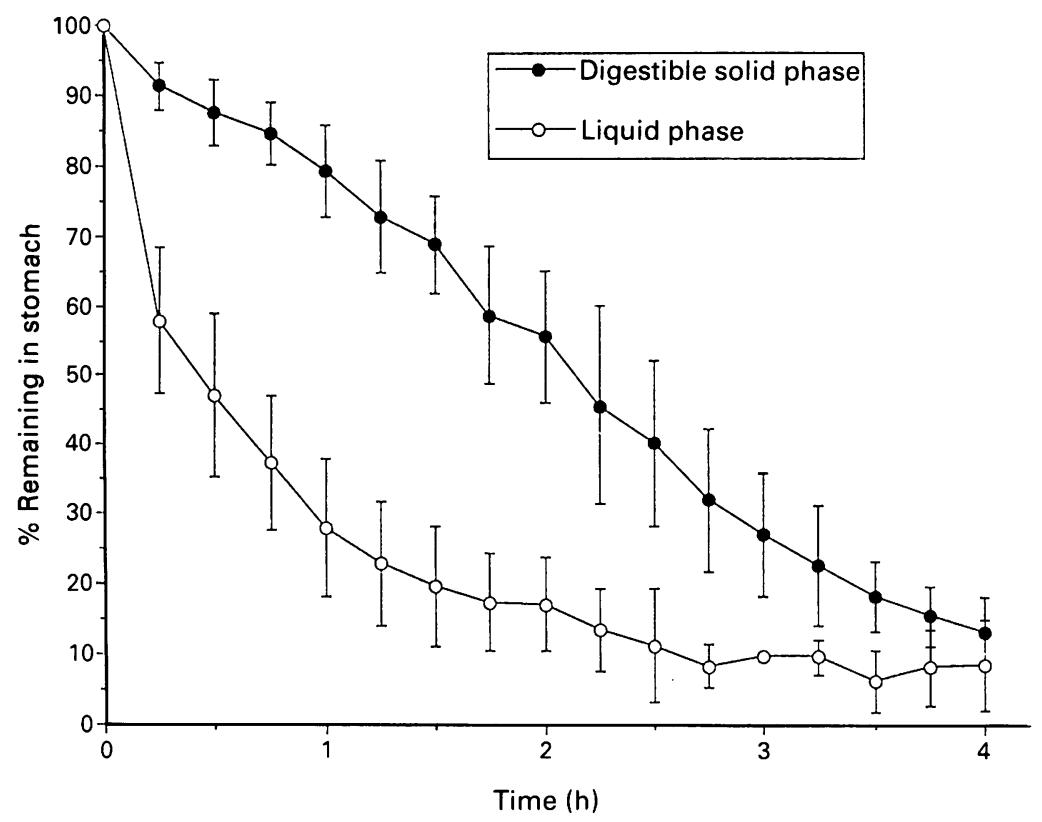

Figure 1: Gastric emptying curves for the digestible and liquid phases of the test meal ( $n=6$; mean $(S E M)$ ).

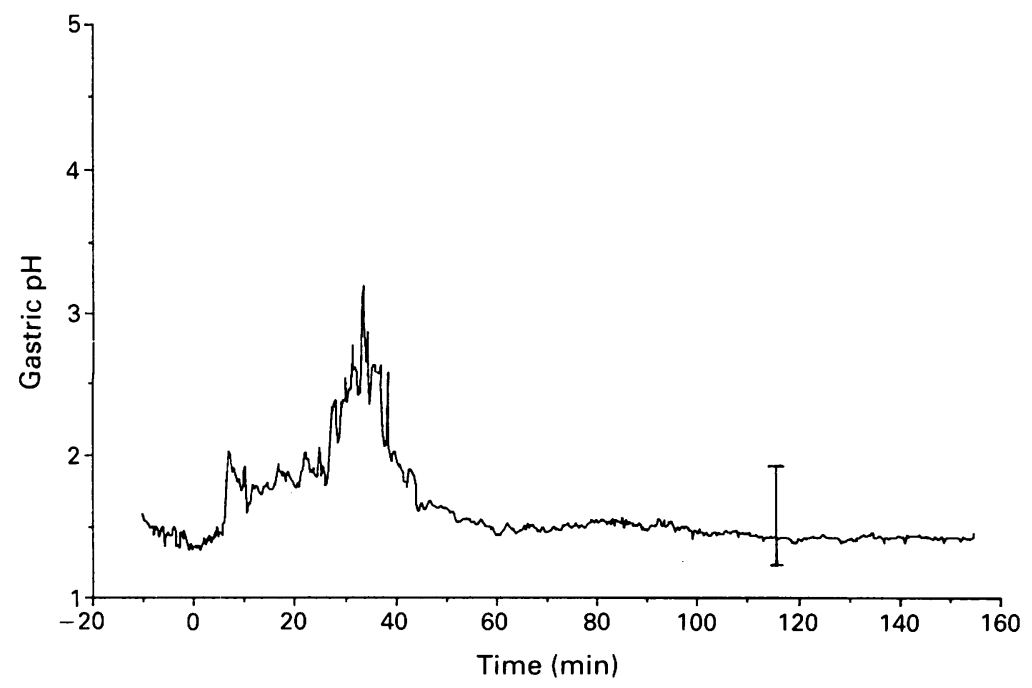

Figure 2: The $p H$ profile caused by the meal in the fundus. The error bar represents a typical SEM over six subjects. removed and the calibration of the $\mathrm{pH}$ probe was checked.

The data from the Memolog recorder were transferred to the computer for analysis. If a drift in calibration of the $\mathrm{pH}$ electrode exceeded $0 \cdot 2$ of a pH unit, a linear correction was made to the $\mathrm{pH}$ data. The refluxed activity was corrected for decay and a baseline rejection was performed, and the area under the $\mathrm{pH}$ peaks calculated. The time for which the oesophageal $\mathrm{pH}$ was below 4 and 5 after the meal was consumed was calculated as a percentage of the total postprandial recording time. Peaks in the refluxed food count data were identified, and backgrounds removed, using a conventional moving average baseline algorithm, and the total amount of refluxed activity present as reflux peaks was calculated.

\section{Results}

\section{IN VITRO STUDIES}

The angular sensitivity of the gamma detector was characterised by its $3 \mathrm{~dB}$ angle. This is the angle through which a source must be moved at constant detector distance to reduce the count to $50 \%$ of that measured with the source on the detector axis. The uncollimated detector had a $3 \mathrm{~dB}$ angle of $75^{\circ}$, and the collimator supplied by the manufacturer with the detector reduced this only to $50^{\circ}$. The $3 \mathrm{~dB}$ angle of the detector was reduced to $20^{\circ}$ using the new collimator. In practice, the main background source that we sought to reject from the refluxed food signal was due to the large pool of activity in the stomach. This material would be approximately $10 \mathrm{~cm}$ from the probe and $10 \mathrm{~cm}$ off its axis, and at this point the detector signal would be reduced to less than $5 \%$ of its value on the detector axis.

The in vitro study showed that the test meal was capable of buffering $0 \cdot 180 \mathrm{~mol}$ of $\mathrm{H}^{+}$ions before the $\mathrm{pH}$ was reduced to $2 \cdot 0$, about basal gastric $\mathrm{pH}$. This required 1 litre of simulated gastric fluid.

\section{IN VIVO STUDIES}

Gastric emptying of liquid and solid phases of the test meal

Figure 1 shows the mean gastric emptying of the test meal. The liquid phase (lemonade) began to empty in a rapid exponential fashion immediately after ingestion. After $0.7(0.09) \mathrm{h}$ (mean (SEM)), half of the liquid phase (T50) had emptied from the stomach. In all subjects, the digestible phase (omelette) of the test meal emptied more slowly than the liquid phase and the mean T50 was $2 \cdot 4(0 \cdot 38) \mathrm{h}$.

\section{Measurement of the $\mathrm{pH}$ in the fundus after the test} meal

Figure 2 shows the mean $\mathrm{pH}$ profile in the fundus. The mean baseline fundal $\mathrm{pH}$ was $1 \cdot 75$ $(0.25)$, and after ingestion of the test meal rose rapidly to $2 \cdot 75(0.75)$ by 10 minutes. It remained at this value for a further 20 minutes before rising again to $3.5(0.5)$ by 30 minutes and then falling back to baseline by $2 \cdot 5$ hours. 


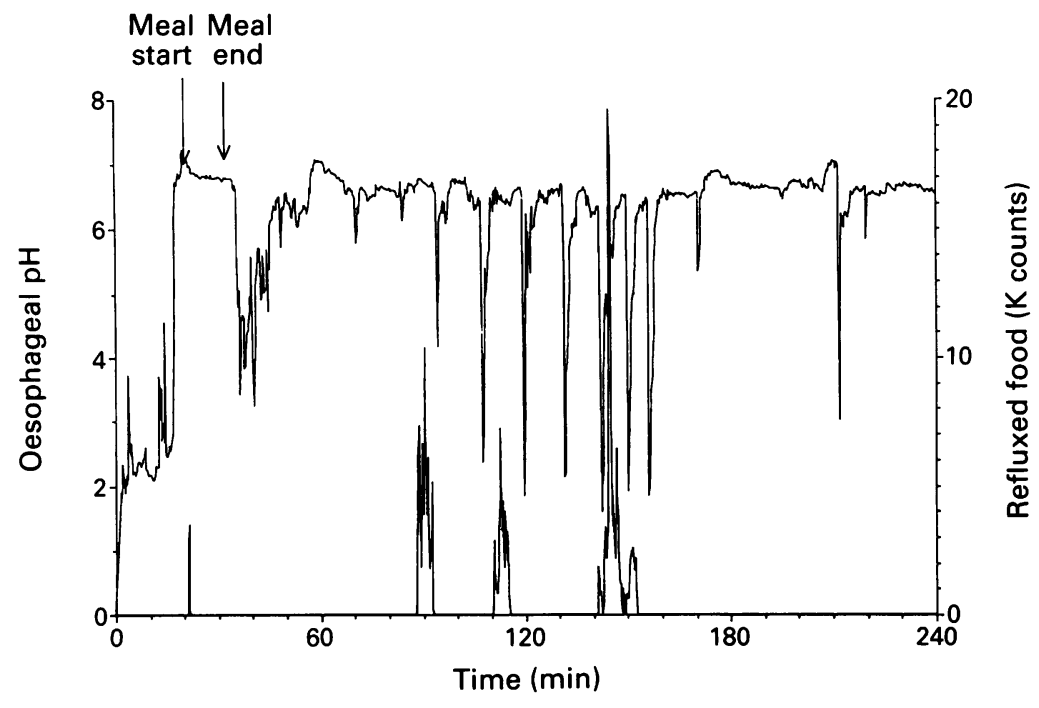

Figure 3: Typical oesophageal pH (upper trace) and count data (lower trace) from a single subject.

\section{pH Monitoring and gamma detection}

The oesophageal $\mathrm{pH}$ and refluxed activity from each subject was plotted against time, and a typical trace is shown in Figure 3. All subjects reported belching after the meal.

Figure 4 shows the indvidual data for the

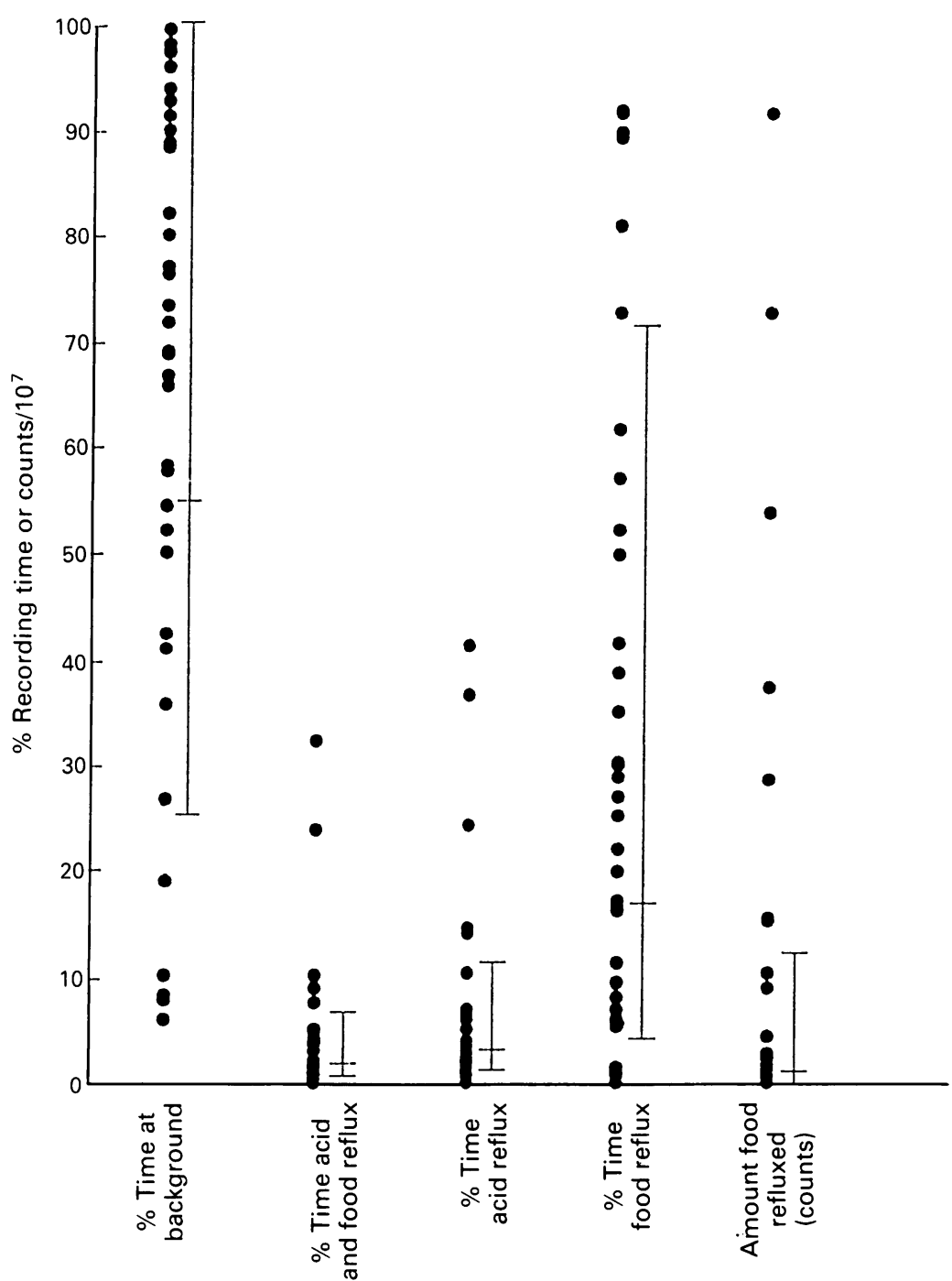

Figure 4: Data from all subjects showing the percentage of time at background, or with actd or food reflux, or both acid and food reflux. Note that the final column (amount of food refluxed) is scaled to the same vertical axis for comparison, and so shows only the comparative variation in this parameter among the subjects. subjects. This represents the percentage time at background - that is, no food or acid reflux, the percentage of time with food reflux or acid reflux, and the percentage for which both food and acid were refluxed. Also shown is the total amount of food refluxed (expressed as counts $/ 10^{7}$ of refluxed activity). These variables were strongly skewed and seemed lognormally distributed, so a log transform was used before calculating their means and deviations.

The oesophageal $\mathrm{pH}$ fell below 4 for $3 \cdot 2+8 \cdot 6 /$ $-2 \cdot 3 \%$ (mean (SD)) of the recording time. Not every reflux event detected, however, by a fall in $\mathrm{pH}$ to less than 4 was seen as an increase in counts because of reflux of food, and vice versa. Food reflux alone occurred for $17 \cdot 8+53 \cdot 2 /-13 \cdot 8 \%$ of the recording time. Simultaneous food and acid reflux occurred for only $0 \cdot 95+5 \cdot 2 /-1 \cdot 2 \%$ of the time. A wide variation among subjects was also seen in the total amount of food refluxed into the oesophagus. This was $(0.95+13.0 /-0.9) \times 10^{\prime}$ counts, corresponding to a rather low mean but with a significant number of higher outliers.

\section{Discussion}

GASTRIC BEHAVIOUR OF THE TEST MEAL

The mean fundal baseline $\mathrm{pH}$ in the subjects was mean (SEM) $1.75(0.25)$, which is in agreement with previous studies. ${ }^{11-13}$ When the peak $\mathrm{pH}$ occurred in the fundus, about $90 \%$ of the solid meal and $50 \%$ of the liquid phase of the meal remained in the stomach. This rapid rise in fundal $\mathrm{pH}$ is due to the buffering effect of the food, largely because of the protein present, and this has been seen in previous experiments with different test meals. ${ }^{113-15}$ The $\mathrm{pH}$ of the test meal when measured in vitro was found to be 4.9 , but this was not achieved in vivo because of the presence of an initial amount of gastric juice, and because of the immediate stimulation of acid production by the food. ${ }^{16}$ Two hours after the meal was ingested, fundal $\mathrm{pH}$ had returned to baseline, but about $50 \%$ of the food solid phase and $20 \%$ of the liquid phase remained. This behaviour is typical of the several meals we have investigated using scintigraphic techniques, and shows that it represents a physiologically relevant test meal.

\section{OESOPHAGEAL PH AND FOOD REFLUX}

The subjects, all of whom had no previous history of reflux disease or gastric disturbances, showed a wide range of behaviour. In general, the amounts of refluxed acid were low, the average person having acid in the oesophagus for about $3 \%$ of the recording time. A significant number of subjects (7/37), however, had food reflux that was outside one standard deviation from the mean, whereas only one of these subjects would have had a positive reflux score by measuring acid alone. The reflux of acid with no corresponding increase in counts suggests that although gastro-oesophageal reflux is stimulated by the presence of a meal, it is not simply acidified food that reaches the oesophagus, but unlabelled gastric acid. This implies incomplete mixing of gastric acid with the food mass. A 
similar finding has been reported using simultaneous gamma scintigraphy, oesophageal $\mathrm{pH}$ monitoring, and manometry in which only $23 \%$ of reflux events were detected by both methods. ${ }^{3}$ In this study scintigraphy detected more reflux events $(61 \%)$ than the $\mathrm{pH}$ monitoring alone $(16 \%)$.

Oesophageal contractions not associated with acid reflux have been reported. ${ }^{17}$ It is possible that these correspond to alkaline reflux, which would not be detected using the gold standard test of intraoesophageal $\mathrm{pH}$ monitoring. There can often be a poor correlation between acid reflux and the degree or type of damage to the oesophagus; for example, there is no significant difference between the extent and patterns of reflux in patients with severe oesophagitis and those who have developed Barrett's oesophagus. ${ }^{18}$ The ability to radiolabel the neutral components of reflux such as food or even bile using technetium-99m labelled hepatioiminodiacetic acid (Tc-99m HIDA) offers exciting opportunities towards the basic understanding of gastro-oesophageal reflux, mechanisms of gastro-oesophageal disease, and the effectiveness of its treatment ${ }^{1920}$ without increasing the discomfort of the patient.

Oesophageal gamma counting for the diagnosis of reflux of neutral materials is a simple, noninvasive technique. The small gamma probe used in our study has the added advantage that the patient is free to have a normal routine while the detector is worn. This technique can provide information to assess reflux in terms of frequency and duration of both acid and alkaline reflux and allows the validation of current theories as to the development of complications in this disease. The technique is also useful in the assessment of the efficacy of anti-reflux treatment because these formulations are of neutral $\mathrm{pH}$ and cannot be discriminated from normal oesophageal $\mathrm{pH}$.

The authors would like to thank Dr Dagmar Long from the Department of Gastroenterology, Leeds Royal Infirmary for the design of the test meal, and Mr Mike Barker from the Department of Medical Physics, Leeds Royal Infirmary for producing the prototype collimator for the cadmium telluride detector. The authors also gratefully acknowledge Dr Robin Spiller for holding the ARSAC licences. This study was financially supported by Reckitt and Colman Products (UK).
1 DeMeester TR, Johnson LF. The evaluation of objective measurements of gastro-oesophageal reflux and their contribution to patient management. Surg Clin N Am 1976; 56: 39-53.

2 Kaul B, Petersen H, Grette K, Erichsen H, Myrvold HE. Scintigraphy, $\mathrm{pH}$ measurement and radiography in the Scintigraphy, $\mathrm{pH}$ measurement and radiography in the
evaluation of gastro-oesophageal reflux. Scand $\mathcal{J}$ Galuation of gastro-oesop

3 Shay SS, Eggli D, Johnson LF. Simultaneous esophageal pH monitoring and scintigraphy during the postprandial period in patients with severe reflux esophagitis. Dig Dis Sci 1991; 36: 558-64.

4 Martins JCR, Issacs PET, Sladen GE, Maisey MN, Edwards S. Gastro-oesophageal reflux scintigraphy compared with $\mathrm{pH}$ probe monitoring. Nucl Med Commun 1984; 5: 201-4.

5 Kaul B, Petersen H, Grette K, Myrvold HE. Reproducibility of gastroesophageal reflux scintigraphy and the standard acid reflux test. Scand $\mathcal{F}$ Gastroenterol 1986; $21: 795-8$.

6 Branicki FJ, Evans DF, Ogilvie AL, Atkinson M, Hardcastle JD. Ambulatory monitoring of oesophageal pH using a portable radiotelemetry system. Gut 1982; 23: 992-8.

7 Washington N, Moss HA, Wilson CG. The use of an ambulatory cadmium telluride detector to monitor postprandial reflux of food. Gut 1988; 29: A1451-2.

8 Velasco N. A simple solid-phase marker for gastric emptying studies. Br F Radiol 1982; 55: 533-4.

9 Martin JL, Beck WJ, McDonald AP, Carlson GM, Mathias JR. Technetium-99m labelled solid phase meal: a quantitative clinical measurement of human gastric emptying. $\mathcal{F} \mathrm{Clin}$ Gastroenterol 1983; 5: 315-9.

10 Washington N, Washington C, Wilson CG. Gastric distribution and residence time of two anti-reflux formulations. International fournal of Pharmaceutics 1987; 39: 163-71.

11 Malagelada J-R, Longstreth GF, Summerskill WHJ, Go VLW. Measurement of gastric functions during digestion of ordinary solid meals in man. Gastroenterology 1976; 70: 20310.

12 Walt RP, Gomes MdFA, Wood EC, Logan IH, Pounder RE. Effect of daily oral omeprazole on 24 hour intragastric acidity. BMF 1983; 287: 12-4.

13 Oversen L, Bendtsen F, Tage-Jensen U, Pedersen NT, Gram $\mathrm{BR}$, Rune SJ. Intraluminal pH in the stomach, duodenum and proximal jejunum in normal subjects and patients with exocrine pancreatic insufficiency. Gastroenterology 1986; 90: exocrine 62 .

14 Berchtold P, Reinhart WH, Niederhauser U, Koller U, Halter $F$. In vitro tests overestimate in vivo neutralixing capacity of antacids in presence of food. Dig Dis Sci 1985; 30: 522-8.

15 Hardy JG, Evans DF, Zaki I, Clark AG, Tonnesen HH, Gamst ON. Evaluation of an enteric coated naproxen tablet using gamme scintigraphy and $\mathrm{pH}$ monitoring. Int $\mathcal{F}$ Pharm 1987; 37: 245-50.

16 Rune SJ. Individual variation in secreting capacity of gastric acid to stimulation with solid food and with histamine. Clin Sci 1967; 32: 443-52.

17 Armstrong D, Emde C, Bumm R, Castiglione F, Cilluffo T, Blum AL. Twenty-four-hour pattern of esophageal motility in asymptomatic volunteers. Dig Dis Sci 1990; 35: 1190-7.

in asymptomatic volunteers. Dig Dis Sci 1990; 35: 1190-7.
18 Parrilla P, Ortiz A, Martinez de Haro L, Aguayo JL, Ramirez $P$. Evaluation of the magnitude of gastro-oesophageal reflux P. Evaluation of the magnitude of gastro-oeso
in Barrett's oesophagus. Gut 1990; 31: 964-7.

19 Washington N. Investigation into the barrier action of an alginate gastric reflux suppressant, Liquid Gaviscon. Drug Investigation 1990; 2: 23-30.

20 Washington $\mathrm{N}$, Greaves JL, Iftikhar SY. A comparison of gastro-oesophageal reflux in volunteers assessed by ambulatory $\mathrm{pH}$ and gamma monitoring after treatment with either Liquid Gaviscon or Algicon Suspension. Aliment Pharmacol Therap 1992; 6: 579-88. 